Project 1024775

\title{
Promoting uranium immobilization by the activities of microbial phophatases
}

\author{
Sobecky, Patricia A. \\ Georgia Institute of Technology
}

\begin{abstract}
RESULTS TO DATE: Patricia Sobecky and Martial Taillefert - Progress Report
\end{abstract}
The following is a summary of progress in our project ?Promoting uranium immobilization by the activities of microbial phosphatases? during the first funded period of the project.

I. The first objective of this project is to determine the relationship of phosphatase activity to metal resistance in subsurface strains and the role of lateral gene transfer (LGT) in dissemination of nonspecific acid phosphatase genes. Nonspecific acid phosphohydrolases are a broad group of secreted microbial phosphatases that function in acidic-to-neutral $\mathrm{pH}$ ranges and utilize a wide range of organophosphate substrates. We have previously shown that PO43- accumulation during growth on a model organophosphorus compound was attributable to the overproduction of alkaline phosphatase by genetically modified subsurface pseudomonads [Powers et al. (2002) FEMS Microbiol. Ecol. 41:115-123]. During this report period, we have extended these results to include indigenous metal resistant subsurface microorganisms cultivated from the Field Research Center (FRC), in Oak Ridge Tennessee. To address this objective we have been conducting phenotypic screening of phosphatase activities of metal resistant (e.g., lead resistant) FRC isolates from our existing culture collection. Phosphatase phenotypes are tested on TPMG medium that provides a visible color change during growth. FRC isolates exhibiting (positive) phosphatase phenotypes are subsequently selected for further molecularbased characterizations (e.g., zymograms). We subsequently tested phosphate liberation of select FRC isolates (lead resistant; Pbr) shown to exhibit cell surface and/or extracellular phosphatase activity. FRC isolates assayed for phosphate liberation were obtained from the background reference site (AG38), Area 2 (AB44) and Area 3 (U26, X43, Y4, Y9 and Y29). Batch experiments to determine whether or not FRC isolates could hydrolyze and release inorganic phosphate from organophosphorus substrates were conducted with numerous strains including several lead resistant Bacillus sp.Y4, Y9-602 and Y29 isolates. During the 168-hr incubation, the lead resistant isolates hydrolyzed approximately $50 \%$ of the organophosphorus compound (glycerol-3-phosphate; G3P). In contrast, a genetically modified control strain, Pseudomonas veronii, containing a constitutively expressed alkaline phosphatase hydrolyzed $72 \%$ of the G3P. Interestingly, strains that were determined to be lead resistant yet exhibiting phosphatase positive phenotypes (e.g., strains AG38, AB44, U26 and X43) hydrolyzed $<0.5 \%$ of the exogenous G3P. Further studies of lead resistant and lead sensitive FRC strains will focus on determining if phosphatase activity is mediated by alkaline phosphatases, non-specific acid phosphatases or polyphosphates.

The analysis of a subset of aerobic heterotrophic FRC isolates cultured from radionuclide and metal contaminated FRC soils and uncontaminated (control) sediments indicated a higher percentage of isolates exhibiting phosphatase phenotypes (i.e., in particular those surmised to be PO43--irrepressible) relative to isolates from the background reference site. A high percentage of strains which exhibited such putatively PO43--irrepressible phosphatase phenotypes were also shown to be resistant to the heavy metals lead and cadmium. During this report period, a gram-negative FRC strain, Y9, identified by $16 \mathrm{~S}$ rDNA to be a Rhanella sp., has been shown, in preliminary experiments, to promote the precipitation of uranium from solution. We are also in the process of developing and optimizing primer sets that will be suitable for amplifying microbial acid phosphatases of the known molecular classes [i.e., classes A, B and C]. Primers (20-mer) sets have been developed and are in the process of being tested with control strains and FRC strains from contaminated soils. Studies to determine the tolerance of the FRC strains to uranium have also been initiated during this report period. Cell viability assays were conducted to determine $U$ tolerance using concentrations ranging from 20 microM to 200 microM uranyl acetate during $\mathrm{pH} 4.0$ incubation. These conditions were designed to reflect the in situ $\mathrm{pH}$ and $\mathrm{U}$ concentrations reported 
for the FRC sites. Preliminary testing indicates that the several of the FRC isolates have an enhanced tolerance to uranyl acetate relative to control strains.

II. Research on the second objective of our project, the determination of phosphatase activities in promoting $U$ immobilized, has progressed during this report period as follows. During this reporting period we have conducted a series of kinetic studies in solutions containing the $Y 9$ isolate and an organophosphorus compound to determine if the phosphatase activity of $Y 9$ would enhance the precipitation of uranium. For these incubations two organophosphorus compounds with different chemical structures were selected to determine how efficiently Y9 can hydrolyze these compounds. Phytic acid (IP6) is a six-member carbon ring with six attached orthophosphate groups which can provide up to twelve coordinate binding sites. Glycerol-3-phosphate (G3P) is a more labile compound with only one orthophosphate attached to a three carbon chain. At neutral pH both molecules are negatively charged providing reactive sites for cationic binding. At high concentrations of UO22+ (300 microM) the solubility of $\mathrm{U}(\mathrm{VI})$ as a function of (a) G3P and (b) IP6 after 24 hour abiotic equilibration was determined. Solubility of $\mathrm{U}(\mathrm{VI})$ in G3P decreases sharply between 100 microM and 500 microM G3P and then increases at high concentrations. $\mathrm{U}(\mathrm{VI})$ is highly insoluble at low concentrations of IP6 and precipitates completely. At higher concentrations of IP6 the solubility of $\mathrm{U}(\mathrm{VI})$ is enhanced, possibly due to increased repulsive negative charges around the uranyl ion. Preliminary results of the kinetic studies indicate that biotic precipitation of uranium may be enhanced in the presence of G3P and phosphatase activity. $\mathrm{U}(\mathrm{VI})$ analysis was determined by laser-induced fluorescence (LIF) with a pyrophosphate/hypophosphite reagent. We have also determined $\mathrm{U}(\mathrm{VI})$-phosphate speciation at equilibrium, predicted by MINEQL+, as a function of phosphate concentration in solution and $\mathrm{pH}$. Specifically, at low $\mathrm{pH}$ and low phosphate concentration, uranium is mainly in the form of the uranyl ion (UO22+). As the concentration of phosphate approaches that of total uranium, highly insoluble uranium phosphate compounds are formed. In excess phosphate, speciation calculations predict dissolution of this mineral and formation of soluble uraniumphosphate complexes. At higher $\mathrm{pH}$, uranium tends to be insoluble at low concentrations of phosphate and soluble, as uranium phosphate complexes, at high phosphate concentrations. The solid phase preferentially formed at $\mathrm{pH}>7$ is a uranium hydroxide mineral. These calculations were performed in the absence of carbonate species to illustrate the effect of phosphate on uranium speciation. The addition of carbonate does not change the behavior of either uranium or phosphate.

III. The third objective of our project is the determination of geochemical parameters affecting uranium phosphate immobilization. For this objective we plan to focus on conditions relevant to the ORNL FRC site. As the FRC sites contain high concentrations of nitrate and a low $\mathrm{pH}$, we will investigate how tolerant subsurface microorganisms are to nitrate and how $\mathrm{pH}$ changes may affect the biomineralization process. Prior to selection of sites for requesting groundwater and soil samples from the FRC we will consult with Dave Watson (Manager; ORNL FRC) for suitable locations (and avoiding sites that have undergone recent biostimulation events). For these experiments, sediments and groundwater from nearby wells will be collected from the FRC sites where characterized bacterial strains were previously isolated. Specifically, we plan to: 1) characterize the chemical composition of the porewaters and sediments; 2) establish that strains isolated from the FRC sites can immobilize $U$ as uranium phosphate precipitate in natural conditions with sediment slurries of the FRC sites; 3 ) determine the kinetics of precipitation of uranium phosphate in sediments from the FRC sites with different nitrate and $\mathrm{pH}$ conditions with or without the addition of U-precipitating FRC strain(s) in the presence of the natural subsurface populations.

DELIVERABLES: Publications and presentations:

Martinez, R.J. and Sobecky, P.A. Subsurface Uranium Immobilization Enhanced by Microbial Phosphatases. Manuscript in preparation.

Sobecky, P., R. Martinez, M. J. Beazley, and M. Taillefert. Promoting Uranium Immobilization by the Activities of Microbial Phosphatases. Poster presentation. Annual NABIR PI meeting, Warrenton, VA March, 2005 
Martinez, R.J., J. R. Elsenbeck, M. Taillefert, P. A. Sobecky. Promoting Uranium Immobilization by the Activities of Microbial Phosphatases. Poster presentation. Annual American Society for Microbiology meeting, Atlanta, GA June, 2005.

Taillefert, M., J.R. Elsenbeck, and P.A. Sobecky. Biomineralization of uranium in the presence of organic phosphorus. Invited presentation, American Chemical Society meeting, San Diego, CA March, 2005.

COLLABORATIONS: Patricia Sobecky, PI, Georgia Institute of Technology - molecular microbial ecology Martial Taillefert, Co-PI, Georgia Institute of Technology - chemical analysis 